\title{
Ryanodine Receptors Are Regulated by the Circadian Clock and Implicated in Gating Photic Entrainment
}

\author{
Karen L. Gamble ${ }^{1}$ and Christopher M. Ciarleglio ${ }^{2}$ \\ ${ }^{1}$ Department of Biological Sciences, Vanderbilt University, Nashville, Tennessee 37235-1634, and ${ }^{2}$ Vanderbilt Kennedy Center, Vanderbilt University, \\ Nashville, Tennessee 37203 \\ Review of Pfeffer et al.
}

In mammals, $24 \mathrm{~h}$ rhythms are orchestrated by the primary circadian pacemaker in the suprachiasmatic nucleus (SCN) of the hypothalamus. Circadian clock function has three components: the mechanism (the "gears"), output (the "hands"), and input (the "set" switch). Rhythmicity is maintained by molecular "gears" in which the CLOCK/BMAL1 heterodimer binds to the promoter regions of Period (Per1 and Per2) and Cryptochrome (Cry1 and Cry2) genes, activating transcription (Fig. $1 A$ ). Negative control of transcription occurs when PER/ CRY dimerize, are phosphorylated by casein kinase I, and translocate into the nucleus, where they inhibit their own transcription. The interaction of these feedback loops results in rhythmic expression of Per and Cry with a peak during the day and a nadir during the night (Fig. 1B) [for review, see Takahashi et al. (2008)]. The output, or "hands" of the clock, is reflected by changes in membrane physiology and in gene expression mediated by the molecular clock gears.

The clock phase is set by the light-dark (LD) cycle through direct input from the retina via the glutamatergic retinohypo-

Received Aug. 5, 2009; accepted Aug. 14, 2009.

This work was supported by National Institutes of Health Grants K99 GM086683 (K.L.G.) and T32 MH64913 and F31 MH080547 (C.M.C.). We thank Shin Yamazaki for insightful comments in the writing of this manuscript.

Correspondence should be addressed to Karen L. Gamble, Department of Biological Sciences, Vanderbilt University, 1210 MRBIII, VUStation B, Box 35-1634, Nashville, TN 37235-1634. E-mail: karen.gamble@vanderbilt.edu.

D01:10.1523/JNEUROSCI.3820-09.2009

Copyright $\odot 2009$ Society for Neuroscience $\quad$ 0270-6474/09/2911717-03\$15.00/0 thalamic tract. NMDA receptor activation in retinorecipient SCN cells allows calcium entry, which activates ryanodine receptors (RyRs) on intracellular calcium stores during the early night. The resulting increase in intracellular calcium levels leads to activation of second messenger pathways and upregulation of Per1 and Per2, and thus sets the clock to an evening phase after an early-night light pulse (a phase delay). A light pulse presented during the late night results in NMDA receptormediated calcium entry, leading to activation of the protein kinase G (PKG)/cGMP pathway and upregulation of Perl and Per2, and thus sets the clock to a morning phase (a phase advance) as illustrated by Pfeffer et al. (2009) (their Fig. 7). For example, RyR activation appears to be necessary for light-induced phase delays of wheel-running behavior in the early night but not for advances in the late night. Conversely, activation of the PKG/cGMP pathway is necessary for glutamate-induced phase advances in SCN firing rate rhythms during the late night but not for delays during the early night (Ding et al., 1998). Although light stimulation during both early and late phases of the night results in upregulation of Per, the ensuing phase shift is different because Per levels are declining in the early night and rising during the late night [for review, see Brown and Piggins (2007)].

Although it is estimated that the molecular clock provides circadian regulation of $\sim 6-10 \%$ of the mammalian genome [1800-3000 genes (Panda et al., 2002)], it is unknown whether the input pathway of the clock is also subject to circadian regulation. Clock regulation of the input pathway would be advantageous because it would allow differential sensitivity to light at various phases of the cycle. Pfeffer et al. (2009) addressed the issue of photic gating in a recent article in The Journal of Neuroscience. To examine the input pathway of circadian clock function independent of the mechanism or output, they used mice that are deficient in BMAL1.

Similar to Bunger et al. (2000), Pfeffer et al. (2009) found that Bmal1-/- mice were behaviorally arrhythmic in constant darkness [Pfeffer et al. (2009), their supplemental Fig. 1]. In addition, the oscillations of Perl and Per 2 mRNA levels in an LD cycle were absent and remained constitutively low [Pfeffer et al. (2009), their Fig. 2a]. To assess the phase response to light, the authors assume that the molecular clock is functioning under LD conditions in Bmal1-1- mice. According to their Figure $2 a$, however, the molecular clock does not appear to cycle even in LD, and thus "phase" has no real meaning. Although defining phase in arrhythmic animals is tenuous, Pfeffer et al. (2009) subjected the mice to a $15 \mathrm{~min}$ light pulse during the early night ( $2 \mathrm{~h}$ after lights off), late night ( $2 \mathrm{~h}$ before lights on), and subjective day (after 42 h of constant darkness) and measured light-induced Per1 and Per 2 mRNA levels $2 \mathrm{~h}$ after the light 

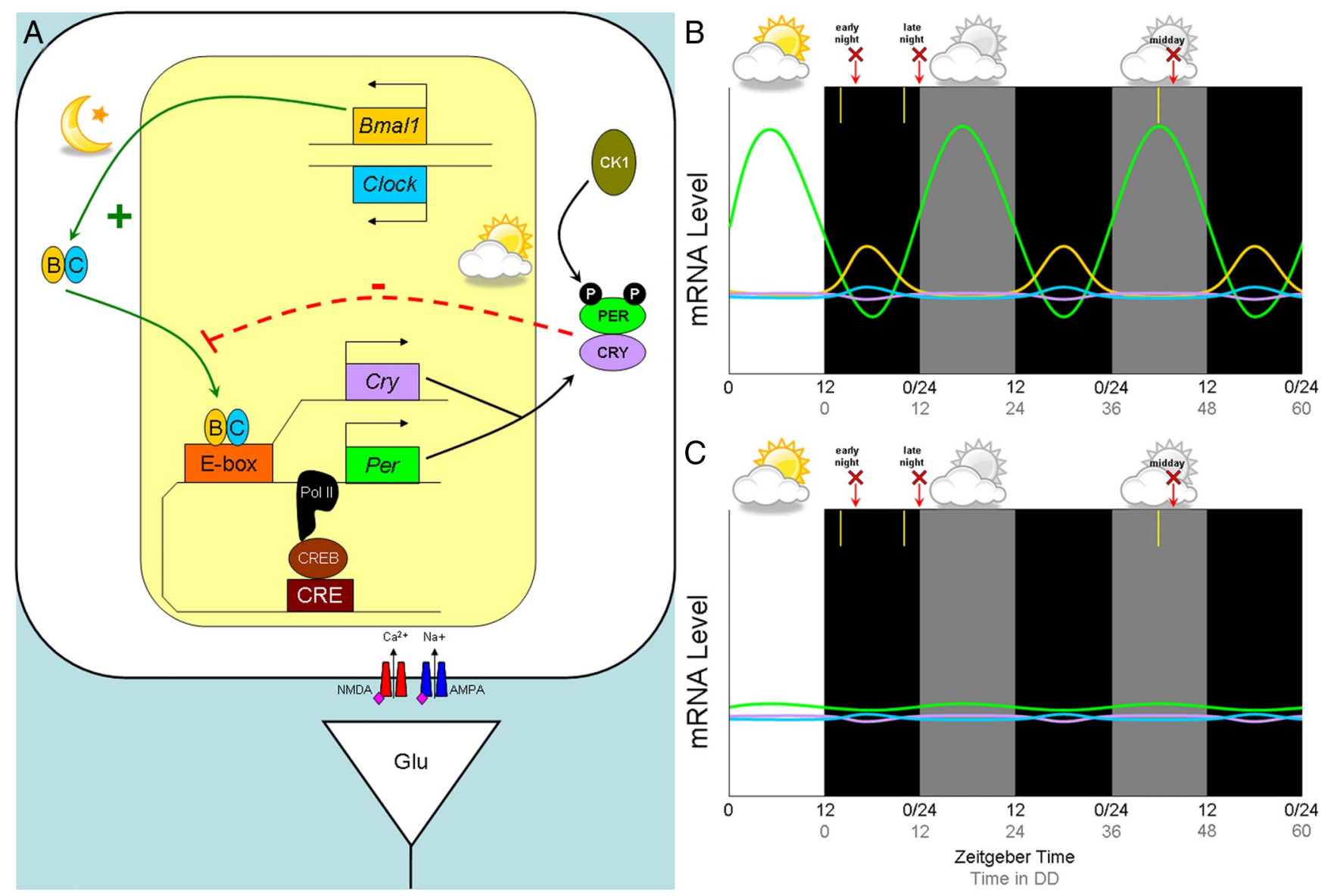

Figure 1. The molecular components of the circadian clock and their circadian expression in a retinorecipient neuron of the $S C N$. $A$, The circadian clock gene/protein network that drives an $\sim 24$ h period contains a positive limb with BMAL1 and CLOCK activating transcription of Per and Cry. PER and CRY proteins are phosphorylated by casein kinase 1 (CK1) and form the negative limb by downregulating their own transcription. $\boldsymbol{B}, \boldsymbol{C}$, Typical expression of generalized circadian genes over time in a wild-type mouse $(\boldsymbol{B})$ and in a $B m a l 1-/-$ mouse $(\boldsymbol{C})$, based on data from Pfeffer et al. (2009). White and black backgrounds represent lights on (daytime) or lights off (night), while a gray background represents "subjective day" in constant darkness. The $y$-axis refers to relative mRNA levels, and the $x$-axis refers to time (in hours), with the black numbers corresponding to zeitgeber time (ZT; where ZT 12 refers to lights off) and the gray numbers corresponding to hours in constant darkness. In the study by Pfeffer et al. (2009), $15 \mathrm{~min}$ light pulses were given at $2 \mathrm{~h}$ after dusk (early night), $2 \mathrm{~h}$ before dawn (late night), or after $42 \mathrm{~h}$ in constant darkness ("subjective day") as indicated by the yellow bars; animals were killed $2 \mathrm{~h}$ after each respective light pulse as indicated by the red arrow and "X." Genes correspond by color to those introduced in $\boldsymbol{A}$.

pulse as indicated in Figure 1, $B$ and $C$. In wild-type SCN, Per1 and Per2 mRNA levels were increased only when light pulses were presented during the early and late night [Pfeffer et al. (2009), their Fig. 1], but not during the "subjective day" (after $42 \mathrm{~h}$ of constant darkness) because the levels were already at a peak [Pfeffer et al. (2009), their Fig. 2b]. In contrast, SCN from Bmal1-/- mice exhibited upregulated Per 1 and Per 2 when pulsed during the late night or "subjective day" [Pfeffer et al. (2009), their Fig. 1b, Fig. 2b], but not during the early night [Pfeffer et al. (2009), their Fig. 1a]. These data are remarkable given that the authors report that a full $12 \mathrm{~h}$ of light during the daytime has no effect on Per expression in Bmal1-/- mice [Pfeffer et al. (2009), their Fig. $2 a$ vs Fig. 1]. These seemingly inconsistent results may simply be due to a lack of resolution in their measure of diurnal regulation, as suggested by the $m P e r 2$ results for $B m a l 1-/-$ mice in their Figure $2 a$.

The disrupted light-induced Per upregulation that occurs during the early night in mice that are deficient in BMAL1 suggests that the input pathway is disrupted only at this time of day, and that BMAL1 deficiency disrupts the circadian regulation of RyRs but not cGMP signaling because light-induced $\mathrm{Per}$ upregulation remains intact during the late night. The authors reported that Ryr2 mRNA and RyR protein levels in the SCN were constitutively low in Bmal1 - / - mice but exhibited a circadian rhythm in wild-type mice [Pfeffer et al. (2009), their Fig. 3a, Fig. 4a]. These data provide the first demonstration that Ryr2s are transcriptionally regulated in wild-type mice by the clock, with mRNA levels peaking during the early subjective day and protein levels peaking $\sim 10 \mathrm{~h}$ later (around dusk).
Pfeffer et al. (2009) compare this temporal sequence of RyR expression to the time lag in Per transcription and translation, but this comparison is limited because the measurements were made in different lighting conditions (constant darkness and a lightdark cycle) and because the frequency of time points (up to every $6 \mathrm{~h}$ for protein) did not allow the necessary resolution. Nevertheless, the time course of Ryr2 transcription suggests that the transcription of this gene may be regulated by the positive limb of the molecular clock; namely, by CLOCK/ BMAL1 activation. Using a Ryr2 promoterdriven luciferase reporter assay in NIH3T2 fibroblasts, the authors elegantly demonstrated that CLOCK and BMAL1 induce transcription of Ryr2 transcriptional activity, which is significantly repressed by the circadian negative regulator, CRY1 [Pfeffer et al. (2009), their Fig. 3c].

Finally, using calcium imaging of SCN neurons from Bmal1-/- and wild-type 
mice, Pfeffer et al. (2009) showed that when clock-driven transcription of RyRs is disrupted, RyR function is also compromised. A significantly larger percentage of SCN cells exhibited increased intracellular calcium levels following bath application of the RyR agonist caffeine in wild-type mice than in Bmal1-/- mice [Pfeffer et al. (2009), their Fig. 5b]. The calcium response to glutamate application was the same in both genotypes. Thus, it appears that in the absence of BMAL1, RyR function is compromised while glutamate receptor function is not. Future experiments could test whether RyR blockade differentially attenuates glutamate-induced calcium increases during the early and late phases of the night.

The Pfeffer et al. (2009) study offers insight into how photic input into the circadian clock is selectively gated, allowing light to reset the phase at certain times of the night but not during the day. This study showed that RyRs undergo circadian regulation, with protein levels elevated during the day and highest at dusk. Thus, these results may explain how the molecular clock regulates the input pathway components during the early night. In addition to affecting the input pathway, it is interesting that RyRs have been implicated in the output of the clock as well (Honma and Honma, 2003; AguilarRoblero et al., 2007; Mercado et al., 2009).

Although the Pfeffer et al. (2009) study has contributed to the field, there are several limitations that necessitate discussion. In addition to the previously mentioned difficulty with showing a phase-specific defect in a system that does not cycle, the effects of developing without a particular gene may contribute to the phenotype of the adult, which is a limitation of any knock-out model. One way to address this caveat would be to examine light-induced Per expression in a Bmal1-/- mouse with a tissue-specific rescue using a tetracyclineresponsive Bmal1 transgene (McDearmon et al., 2006). Finally, the authors suggest that the late-night photic pathway remains intact in Bmal1 - /- mice because the PKG pathway is unaltered. However, previous research has shown that cGMP levels and PKG activity are also regulated by the circadian clock and exhibit a peak during the late night (Tischkau et al., 2003). In a mouse model in which the circadian clock mechanism is severely disrupted, it is likely that the PKG pathway is also compromised. Future studies could examine diurnal variation (in LD cycles) in cGMP levels and PKG activity in Bmal1-/- mice.

In conclusion, this research by Pfeffer et al. (2009) showed that RyRs are transcriptionally regulated by the positive elements of the circadian clock, CLOCK/ BMAL1. In recent years, circadian clocks have been discovered throughout the body and other areas of the brain (Takahashi et al., 2008). Moreover, clock-driven gene regulation is tissue specific such that the same genes regulated in one tissue may be a different set of clock-controlled genes in another (Panda et al., 2002). Given the importance of intracellular calcium regulation in many other cellular and neural processes, future studies should consider CLOCK/BMAL1-regulated transcription of RyRs and circadian variation in other neural systems.

\section{References}

Aguilar-Roblero R, Mercado C, Alamilla J, Laville A, Díaz-Muñoz M (2007) Ryanodine receptor $\mathrm{Ca} 2+$-release channels are an output pathway for the circadian clock in the rat suprachiasmatic nuclei. Eur J Neurosci 26: 575-582.
Brown TM, Piggins HD (2007) Electrophysiology of the suprachiasmatic circadian clock. Prog Neurobiol 82:229-255.

Bunger MK, Wilsbacher LD, Moran SM, Clendenin C, Radcliffe LA, Hogenesch JB, Simon MC, Takahashi JS, Bradfield CA (2000) Mop3 is an essential component of the master circadian pacemaker in mammals. Cell 103: 1009-1017.

Ding JM, Buchanan GF, Tischkau SA, Chen D, Kuriashkina L, Faiman LE, Alster JM, McPherson PS, Campbell KP, Gillette MU (1998) A neuronal ryanodine receptor mediates light-induced phase delays of the circadian clock. Nature 394:381-384.

Honma S, Honma K (2003) The biological clock: $\mathrm{Ca} 2+$ links the pendulum to the hands. Trends Neurosci 26:650-653.

McDearmon EL, Patel KN, Ko CH, Walisser JA, Schook AC, Chong JL, Wilsbacher LD, Song EJ, Hong HK, Bradfield CA, Takahashi JS (2006) Dissecting the functions of the mammalian clock protein BMAL1 by tissue-specific rescue in mice. Science 314:1304-1308.

Mercado C, Díaz-Muñoz M, Alamilla J, Valderrama K, Morales-Tlalpan V, Aguilar-Roblero R (2009) Ryanodine-sensitive intracellular Ca2+ channels in rat suprachiasmatic nuclei are required for circadian clock control of behavior. J Biol Rhythms 24:203-210.

Panda S, Antoch MP, Miller BH, Su AI, Schook AB, Straume M, Schultz PG, Kay SA, Takahashi JS, Hogenesch JB (2002) Coordinated transcription of key pathways in the mouse by the circadian clock. Cell 109:307-320.

Pfeffer M, Müller CM, Mordel J, Meissl H, Ansari N, Deller T, Korf HW, von Gall C (2009) The mammalian molecular clockwork controls rhythmic expression of its own input pathway components. J Neurosci 29:6114-6123.

Takahashi JS, Hong HK, Ko CH, McDearmon EL (2008) The genetics of mammalian circadian order and disorder: implications for physiology and disease. Nat Rev Genet 9:764-775.

Tischkau SA, Weber ET, Abbott SM, Mitchell JW, Gillette MU (2003) Circadian clock-controlled regulation of cGMP-protein kinase $\mathrm{G}$ in the nocturnal domain. J Neurosci 23:75437550 . 